\title{
Oral Appliance Therapy Should be Prescribed as a First-Line Therapy for OSA during the COVID-19 Pandemic
}

\author{
David Schwartz, DDS ${ }^{1}$; Nancy Addy, DDS²; Mitchell Levine, DMD³; Harold Smith, DDS ${ }^{4}$
}

${ }^{1}$ North Shore Family Dentistry, Skokie, IL; ${ }^{2}$ Snoring and Sleep Apnea Dental Treatment Center, Leawood, KS; ${ }^{3}$ Department of Orthodontics, University of Tennessee Health Science Center, Memphis, Tennessee; ${ }^{4}$ Dental Sleep Medicine of Indiana, Indianapolis, Indiana

It is the position of the American Academy of Dental Sleep Medicine (AADSM) that oral appliance therapy (OAT) should be prescribed as a first-line therapy for the treatment of obstructive sleep apnea (OSA) during the COVID-19 pandemic.

This recommendation is being made based on the following information:

- The American Academy of Sleep Medicine (AASM) has indicated that PAP therapy potentially exposes individuals, especially those in proximity to the patient, to increased risk of transmission of COVID-19. ${ }^{1}$

- A number of studies have found evidence that COVID-19 can remain suspended in the air in aerosol particles. ${ }^{2}$

- It is unknown whether it is possible for patients to be re-infected from re-using PAP tubing, filters, and/or masks.

- There have been reported shortages of distilled water and other supplies necessary for optimal use of PAP machines.

- Oral appliances do not generate aerosols and can be easily disinfected by patients to kill the COVID-19 virus.

According to the AADSM and AASM clinical practice guideline, oral appliances should be prescribed for adult patients who prefer alternate therapy to PAP. ${ }^{3}$ During this pandemic, it is reasonable to assume that patients would prefer a therapy that both treats their OSA and does not increase the risk of COVID-19 transmission.

Oral appliance therapy is an effective treatment for OSA $^{3}$ without increased risks for transmitting COVID-19. Patients who prefer OAT may be more likely to adhere to treatment, if they are confident that their therapy is not putting those in their household at an increased risk for COVID-19 exposure.

\section{CITATION}

Schwartz D, Addy N, Levine M, Smith H. Oral appliance therapy should be prescribed as a first-line therapy for OSA during the COVID-19 pandemic. J Dent Sleep Med. 2020;7(3).

\section{REFERENCES}

1. COVID-19: FAQs for Sleep Clinicians. https://aasm.org/covid-19resources/covid-19-faq/. Accessed May 8, 2020.

2. Liu, Y., Ning, Z., Chen, Y. et al. Aerodynamic analysis of SARSCoV-2 in two Wuhan hospitals. Nature (2020). https://doi.org/10.1038/s41586-020-2271-3

3. Ramar K, Dort LC, et al. Clinical Practice Guideline for the Treatment of Obstructive Sleep Apnea and Snoring with Oral Appliance Therapy: An Update for 2015 An American Academy of Sleep Medicine and American Academy of Dental Sleep Medicine Clinical Practice Guideline. J Dent Sleep Med. 2015;2(3). doi:10.15331/jdsm.4868

\section{SUBMISSION AND CORRESPONDENCE INFORMATION}

\section{Submitted in final revised form May 14, 2020.}

Address correspondence to: David Schwartz, DDS; Email: dschwartz@aadsm.org

\section{DISCLOSURE STATEMENT}

All authors are members of the AADSM Board of Directors Executive Committee. Dr. Schwartz is a consultant of ResMed and has conducted lectures and speaking engagements for SomnoMed and ProSomnus Sleep Technologies. 